\title{
Seroprevalence of Toxoplasma gondii and rubella among pregnant women in central Turkey
}

\author{
Çelen Şevki ${ }^{1}$, Sargın Ayla ${ }^{1}$, Çitil Ayşe ${ }^{2 \star}$, Saygan Sibel $^{3}$, Ünlü Serpil ${ }^{4}$, Danışman Nuri ${ }^{1}$ and \\ Seçkin Berna ${ }^{1}$
}

\footnotetext{
${ }^{1}$ Obstetrics and Gynecology Zekai Tahir Burak Womens' Health Education and Research Hospital, Ankara, Turkey. ${ }^{2}$ Obstetrics and Gynecology, Zekai Tahir Burak Womens' Health Education and Research Hospital, Ankara, Turkey. ${ }^{3}$ Microbiology and Clinical Microbiology Zekai Tahir Burak Womens' Health Education and Research Hospital, Ankara, Turkey.

${ }^{4}$ Infectious Disease and Clinical Microbiology Zekai Tahir Burak Womens' Health Education and Research Hospital, Ankara, Turkey.
}

Accepted 26 April, 2013

\begin{abstract}
Infection with Toxoplasma gondii (T. gondii), when acquired in pregnancy, can lead to fetal infection, which may ultimately result in the loss of the fetus or in lesions involving the brain and the eyes. Rubella, when acquired in the first 12 weeks of pregnancy, is associated with a nearly $90 \%$ risk of congenital malformations. The aim of this study was to assess the seroprevalence of $T$. gondii and rubella among pregnant women at a tertiary referral hospital in central Turkey and to evaluate the use of our results for the prevention of congenital rubella syndrome (CRS) and congenital toxoplasmosis (CT). Data were obtained from the test results of sera collected from pregnant women during their first routine antenatal visit between 2008-2010. The results of the TORCH serology and avidity tests were evaluated. Age-specific subgroups were arranged for the rubella and $T$. gondii antibodies, and the distribution of seropositivity among the subgroups was assessed. Serum samples were evaluated for rubella $(n=31385)$ and $T$. gondii $(n=30863) \lg G$, $\lg M$, and $\lg G$ avidity. The seropositivity of antibodies for T. gondii was $7869(25.5 \%)$ and $83(0.3 \%)$ for $\operatorname{lgG}$ and $\operatorname{lgM}$, respectively. For rubella, the seropositivity was $29478(93.9 \%)$ and $124(0.4 \%)$ for IgG and IgM, respectively. In cases with both IgM and IgG, seropositivity avidity tests were performed. A high avidity of IgG antibodies was detected in $95(76.6 \%)$ rubella cases and $30(36.1 \%)$ T.gondii cases. Seropositivity for $T$. gondii increased significantly with age, while rubella seropositivity was high in all of the age-specific subgroups. In conclusion $T$. gondii and rubella are common causes of severe congenital infection. Widespread preventive antenatal measures and screening may help to protect against these teratogenic agents. Due to high seroprevalence of rubella, vaccination programs during childhood and preconceptional periods rather than antenatal screening appear to be more efficient for eliminating CRS. Avoidance of raw meat consumption, proper disposal of the fecal material of domestic animals, and elimination of oocysts from raw food are the main measures to prevent infection with $T$. gondii. Antenatal screening for toxoplasmosis is warranted, considering the low seroprevalence and serious fetal hazards of congenital infection.
\end{abstract}

Key words: Toxoplasma gondii, rubella, seroprevalence, avidity.

\section{INTRODUCTION}

Pregnancy is an extraordinary period in a woman's life, with intense physiological and psychological impacts. 
Almost every system in the body is structurally or functionally altered. During pregnancy, the immune system is suppressed, which makes individuals more susceptible to infections. One of the leading causes of perinatal mortality in developing countries is congenital infection. $T$. gondii and rubella are teratogenic agents with similar effects. These two microorganisms can cause severe fetal damage when acquired during pregnancy. Antenatal screening tests have an important role in the diagnosis of these infections.

The rubella virus is a rubivirus from the Togaviridae family. It is a single-stranded RNA virus enveloped by an icosahedral symmetrical capsule and causes infections only in humans. Clinically, it manifests with mild measleslike symptoms. During the prodromal period, fever and flu-like symptoms accompanied by a maculopapular rash are common. The transplacental transmission of the virus during early gestation results in congenital defects. Embryonic cells infected with the rubella virus display chromosomal fragmentations and an inhibition of mitosis (Gladwin and Trattler, 2004).

Rubella predominantly occurs during childhood and adolescence. The incubation period is generally 12-19 days, and the infection commonly has an asymptomatic course. Rash, malaise, fever, conjunctivitis and generalized lymphadenopathy are the most common symptoms in affected patients. Particularly in young women, a selflimiting arthritis may occur. Thrombocytopenic purpura, encephalitis and orchitis are other rare complications.

Serologic tests are widely utilized for the detection of rubella infections (Mann et al., 1981). Culturing and realtime polymerase chain reaction (RT-PCR) is other diagnostic tools. Particularly in suspected cases of congenital rubella infection, RT-PCR is used as a diagnostic tool for amniotic fluid, fetal blood or chorionic villus samples (Tang et al., 2003).

If the maternal infection occurs during early gestation, the probability of severe CRS is highly increased. During the first trimester, fetal infection occurs in more than $80 \%$ of cases of infected mothers (Tang et al., 2003), and more than $70 \%$ of the infected fetuses display signs and symptoms of CRS (Bar-Oz et al., 2004). In addition, maternal rubella infections may result in miscarriages, blighted ovum and stillbirths. The risk of CRS decreases with advancing gestational age; a risk of $55 \%$ at the end of the first trimester declines to $25 \%$ at the end of the second trimester. Fetuses infected with rubella do not always develop CRS. Ultrasonography is a useful tool to identify affected fetuses; however, it can fail to detect accompanying anomalies in a significant number of cases. Deafness, ocular defects, including cataracts and retinopathy, and central nervous system or cardiac malformations are frequently detected anomalies. Other common findings are growth retardation, glaucoma, microphthalmia, microcephaly, cerebral palsy, mental retardation, thrombocytopenic purpura, hepatosplenomegaly and fetal death. Infected infants can shed the virus for one year after birth. Nearly one-third of infants who are asymptomatic at birth develop long-term complications, such as type 1 diabetes mellitus and progressive panencephalitis (Cherry, 2004).

The primary route of preventing CRS is vaccination prior to gestation. If preconceptional counseling has not been provided, pregnant women should be screened for rubella during their first antenatal visit (MMWR, 2001). In the case of preconceptional vaccination, women should be informed not to conceive for at least one month after vaccination. Susceptible pregnant women should be educated to avoid exposure to rubella infection during gestation. Live attenuated rubella vaccine is not recommended for pregnant women, but susceptible women should be vaccinated postpartum. Vaccination does not interfere with breast-feeding.

$T$. gondii is an obligatory intracellular protozoan with three life forms: Trophozoite, cyst and oocyst. T. gondii sexually reproduces only in cats and is excreted in the form of infected cysts in the feces. Cats are the only known reservoir for oocysts. Many organisms are susceptible to infection with $T$. gondii. Humans are infected with the cystic form through the gastrointestinal system via contaminated food or the consumption of raw or undercooked meat. Cats are the main source of infection in humans. Cysts infect mainly the cells of the central nervous system and muscles and are disintegrated with heat (Egerman and Beazley, 1998). Transplacental fetal infection occurs as a result of the primary infection of pregnant women. CT does not occur in individuals who have been previously exposed and acquired immunity to toxoplasmosis. The severity of the fetal infection is inversely proportional to the gestational age. Transplacental transmission of the infection is much higher during the third trimester, and CT is more common during this period. In early gestation, the risk of transplacental transmission is lower, but the risk of fetal damage is severe, and fetal death is quite common. Most of the fetuses with CT are asymptomatic at birth, and $40 \%$ of the infants become symptomatic in time. Symptomatic infants may have a rash, chorioretinitis, blindness, convulsions, mental retardation, microcephaly or encephalitis (Egerman and Beazley, 1998). Asymptomatic infants may later develop retinal inflammation and chorioretinitis with eventual blindness due to reactivation. CT may also result in abortus, stillbirth or preterm labor.

The diagnosis of toxoplasmosis can be completed using serologic or histologic methods, but the serologic tests are not well-standardized. If initial test results indicate acute toxoplasmosis, the tests should be confirmed in a referral laboratory (Egerman and Beazley, 1998). The best way to confirm the diagnosis of CT is the detection of toxoplasma DNA in the amniotic fluid with PCR (Romand et al., 2001). Acute toxoplasmosis should be treated in immunocompromised individuals and pregnant women. Treatment of pregnant women reduces the risk of CT and long-term sequelaes of the infection. The effi- 
Table 1. Distribution of immunglobulins for rubella Infection.

\begin{tabular}{lccccc}
\hline \multirow{2}{*}{ Age } & \multicolumn{2}{c}{ Rubella IgG } & \multicolumn{2}{c}{ Rubella IgM } & \multirow{2}{*}{ Total } \\
\cline { 2 - 5 } & Positive & Negative & Positive & Negative & \\
\hline$\leq 20$ & $2341(95.7 \%)$ & $104(4.3 \%)$ & $9(0.4 \%)$ & $2436(99.6 \%)$ & 2445 \\
$21-25$ & $7982(94.9 \%)$ & $425(5.1 \%)$ & $35(0.4 \%)$ & $8372(99.6 \%)$ & 8407 \\
$26-30$ & $9403(93.7 \%)$ & $628(6.3 \%)$ & $40(0.4 \%)$ & $9991(99.6 \%)$ & 10031 \\
$31-35$ & $6207(92.9 \%)$ & $473(7.1 \%)$ & $24(0.3 \%)$ & $6656(99.7 \%)$ & 6680 \\
$>35$ & $3545(92.7 \%)$ & $277(7.3 \%)$ & $16(0.5 \%)$ & $3806(99.5 \%)$ & 3822 \\
Total & $29478(93.9 \%)$ & $1907(6.1 \%)$ & $124(0.4 \%)$ & $31261(99.6 \%)$ & 31385 \\
\hline
\end{tabular}

Table 2. Distribution of immunglobulins for toxoplasmosis.

\begin{tabular}{cccccc}
\hline \multirow{2}{*}{ Age } & \multicolumn{2}{c}{ ToxoplasmosislgG } & \multicolumn{2}{c}{ ToxoplasmosislgM } & \multirow{2}{*}{ Total } \\
\cline { 2 - 5 } & Positive & Negative & Positive & Negative & \\
\hline$\leq 20$ & $453(18.7 \%)$ & $1971(81.3 \%)$ & $8(0.4 \%)$ & $2416(99.6 \%)$ & 2424 \\
$21-25$ & $1718(20.9 \%)$ & $6521(79.1 \%)$ & $24(0.3 \%)$ & $8215(99.7 \%)$ & 8239 \\
$26-30$ & $2290(23.4 \%)$ & $7481(76.6 \%)$ & $32(0.3 \%)$ & $9739(99.7 \%)$ & 9771 \\
$31-35$ & $1954(29.3 \%)$ & $4708(70.7 \%)$ & $13(0.2 \%)$ & $6649(99.8 \%)$ & 6662 \\
$>35$ & $1454(38.6 \%)$ & $2313(61.4 \%)$ & $6(0.2 \%)$ & $3761(99.8 \%)$ & 3767 \\
Total & $7869(25.5 \%)$ & $22994(74.5 \%)$ & $83(0.3 \%)$ & $30780(99.7 \%)$ & 30863 \\
\hline
\end{tabular}

ciency of intrauterine treatment seems to be comparable to early treatment of the newborn(Foulon et al., 1999).

The aim of this study was to assess the seroprevalence of $T$. gondii and rubella among pregnant women at a tertiary referral hospital in central Turkey and to evaluate current methods for the prevention of CRS and CT.

\section{MATERIALS AND METHODS}

The Zekai Tahir Burak Women Health Education and Research Hospital is one of the largest referral centers in the field of obstetrics and gynecology in Turkey. Approximately 20,000 pregnant women per year are treated in the clinic. Women routinely undergo TORCH screening during their first antenatal visit. We designed a retrospective study that included all of the pregnant women who had antenatal TORCH screening tests at our hospital from 2008 to 2010 . A total of 30,863 pregnant women for T. gondii and 31,385 for rubella were reviewed at different ages and gestational weeks. For the screening test, $8-10 \mathrm{ml}$ of venous blood was taken under sterile conditions from each woman. The antibodies were investigated in the sera using a chemiluminescent immuneassay (CLIA) (LIAISON, DiaSorin S.p.A, Italy) technique. The cutoff values were $8.8 \mathrm{IU} / \mathrm{ml}$ and $11 \mathrm{IU} / \mathrm{ml}$ for $T$. gondii and rubella, respectively. No patient was excluded. If both $\lg G$ and $\lg M$ were positive, $\lg G$ avidity tests were performed. The $\lg G(-)$ but $\lg M(+)$ patients were evaluated again after 2-3 weeks. If only IgM was positive again, it was considered as a nonspecific antibody. If both IgM and IgG were positive after 2-3 weeks, prenatal or postnatal diagnosis was performed.

\section{RESULTS}

During the study period, a total of 31,385 and 30,863 pregnant women were screened for rubella and T. gondii, respectively. The seropositivities of the IgG antibody for T.gondii and rubella were 7,869 (25.5\%) and 29,478 $(93.9 \%)$, respectively. The seropositivities of the $\operatorname{lgM}$ antibody for T.gondii and rubella were $83(0.3 \%)$ and 124 $(0.4 \%)$, respectively. The distribution of the rubella and $T$. gondii IgG antibody titers through the age groups are shown in Tables 1 and 2 . The rubella IgG positivity was observed to be high in all of the age groups (Table 1). The $T$. gondii $\operatorname{lgG}$ positivity increased with increasing age. In pregnant women under the age of 20 , the T.gondii IgG positivity was $18.7 \%$, while the IgG positivity in pregnant women over age 35 was $38.6 \%$ (Table 2).

Avidity tests were recommended for patients whose $\lg \mathrm{M}$ and $\lg \mathrm{G}$ antibody results were both positive. Avidity tests were performed in 95 out of 115 patients who were positive for rubella $\lg M$ and $\lg G$, and high avidity was found in all of the tested cases. Both toxopasma IgM and IgG were positive in 55 out of 69 cases in whom we utilized avidity tests. In 25 cases (45.5\%), a low avidity was found. Table 3 shows the values of $\lg G$ avidity of rubella and toxoplasmosis cases.

\section{DISCUSSION}

Maternal rubella infection and toxoplasmosis pose risks to the fetus and the newborn, particularly when acquired in early gestation. When susceptible women are exposed to these agents, congenital defects, affecting almost all of the organ systems of the fetus, known as CRS and toxoplasmosis occurs. 
Table 3. Results of avidity tests using IgG for toxoplasmosis and rubella infection.

\begin{tabular}{lccccc}
\hline \multirow{2}{*}{ Parameter } & \multicolumn{3}{c}{ Avidity of IgG } & \multirow{2}{*}{ Total } \\
\cline { 2 - 5 } & IgG-negative & Not utilized & High & Low & \\
\hline Rubella IgM-positive & 9 & 20 & $95(100 \%)$ & - & 124 \\
Toxoplasmosis IgM-positive & 14 & 14 & $30(54.5 \%)$ & $25(45.5 \%)$ & 83 \\
\hline
\end{tabular}

To estimate the risk of CRS, we must know the susceptibility of the community, particularly pregnant women, to the rubella virus. In Turkey, there are few studies of infection prevalence with a limited number of patients. Because of this lack of data, an investigation of prevalence among pregnant women is worthwhile.

Worldwide, there is considerable variation in the prevalence of rubella antibodies among women of childbearing age. Seroprevalence is higher in European women (93.2\%) than in women of African (86.7\%) and Asian origin (78.4\%) (Lever et al., 1987). In our study among pregnant women in Turkey, the seroprevalence was similar to that of European women. A total of $93.9 \%$ of the women in the study group had previously been exposed to the infection, and only $6.1 \%$ were susceptible. Our results are also in accordance with seropositivity rates from Iran (96\%), Saudi Arabia (91.1\%) and Egypt (92.2\%) (Doroudchi et al., 2001; Hossain, 1989; Younes et al., 1991).

When the seropositivities among different age groups were evaluated, we did not observe any differences among age groups in the pregnant population.

In our study, we detected a low rate of susceptibility $(6.1 \%)$ to rubella infection among pregnant women. Community-based perinatal investigations are required to ascertain if CRS is a major health issue in a country. In the last decade in Turkey, very few community-based studies among childbearing women have been reported. Akşit et al. (1999) reported rubella seropositivities of 89.7 and $91.6 \%$ for age groups of $15-29$ and $20-29$ years, respectively. Şeker et al. (2004) detected $100 \%$ seropositivity among a 20-41 year age group in the northwest region of İstanbul. For the Ankara region, Cengiz et al. (2005) reported a seropositivity rate of $86.5 \%$ in 2005. Yücel et al. (2002) reported a seropositivity of $93.6 \%$ for the same geographic region in 2002 . Their findings were consistent with our results.

In Turkey, the mumps-measles-rubella (MMR) vaccine has been part of the national vaccination program since 2006 . There may be cases in which the vaccine fails or the protective level of antigens declines in a few years. For this reason, some researchers recommend screening for rubella antibodies among women of childbearing age (Uyar et al., 2008).

It seems that the prevention of CRS may be possible only through efficient vaccination. The World Health Organization (WHO) reported various strategies for the prevention of CRS. These strategies include providing direct protection to women and schoolgirls (a selective vaccination strategy), vaccinating boys and girls to provide indirect protection by reducing the transmission of rubella virus infections (a universal vaccination strategy) and a combination of these two approaches (a combined strategy) (Robertson et al., 1997). Considering that routine MMR vaccination has been implemented since 2006 in Turkey, the combination strategy seems to be the best option to prevent CRS in Turkey.

Worldwide, there is great diversity in the seroprevalence of $T$. gondii reported. The epidemiology of toxoplasmosis is documented in many countries, but a nationwide epidemiological investigation does not exist for Turkey. There are institution-based, retrospective studies with small numbers of patients. Because our hospital is a referral center providing antenatal care for the Ankara region and all of Central Anatolia and a huge number of patients are admitted to our clinic, we consider our results to be a more accurate indicator of the epidemiology of this infection in Turkey.

The seroprevalence of toxoplasmosis is related to factors including socioeconomic status, age, dietary habits, and rural or urban settlement. In a recent study with sera from pregnant women living in northeastern China, the two main risk factors related to $T$. gondii infection were the care of pet animals and the consumption of raw meat (Liu et al., 2009). In our study, seroprevalence increased with increasing age, but there are studies reporting similar seroprevalence values among different age groups (Topuzoglu et al., 2005).

In the first trimester, the risk of congenital infection with T.gondii is $10-15 \%$, and fetal effects can be disastrous. During the second and third trimesters, the probability of fetal infection increases up to $68 \%$, but the fetal effects are milder (Remington et al., 2006). Ocular lesions, which may cause blindness, are the most common long-term sequelae of toxoplasmosis. It has been demonstrated in several studies that chorioretinitis and cerebral lesions are more common in cases with lack of antenatal screening and treatment (Wallon et al., 2004). When seroconversion occurs in a pregnant individual, spiramycin is prescribed. Although its effect on vertical transmission is controversial, all of the patients diagnosed in this manner should be offered antibiotherapy.In cases with positive amniotic fluid PCR results, a combination of pyrimethamine and sulfonamide is substituted instead of spiramycin. This combination is believed to prevent severe congenital sequelae (Thiebaut et al., 2007). In France, where the infection is relatively common, the surveillance 
system records only the lesions apparent at the time of birth, although the actual size of the damage related to congenital toxoplasmosis can only be determined with long-term follow-up of the infants. Infants born with congenital infection carry a risk of ocular lesions leading to blindness during childhood and adolescence even if they appear asymptomatic at birth (Gras et al., 2005).

The surveillance system for congenital toxoplasmosis varies in different European countries, and principally, it depends on the prevalence in that region. We do not have a routine national screening program for $T$. gondii and rubella infections in Turkey. Because the cost-effectiveness of screening is not yet clear, these tests are not a routine element of nationwide antenatal care.

In our study, the seropositivity of $T$. gondii was determined to be $25.5 \%$. Due to the low seropositivity, antenatal screening may be beneficial for the prevention of congenital toxoplasmosis in the studied region.

The consumption of well-cooked meat, the proper handling and disposal of fecal material from pet cats, and the elimination of oocysts from raw-consumed food are the most important measures for prevention of $T$. gondii infections. In a recent study from northeastern China, taking care of pet animals and handling raw meat were the two main factors causing toxoplasmosis (Liu et al., 2009). The education of women of childbearing age regarding prevention of the infection is a major public health issue.

\section{Conclusion}

There is no national routine-screening program for toxoplasmosis and rubella infection in Turkey. Congenital toxoplasmosis is not screened in many European countries due to the lack of evidence of the cost-effectiveness of screening. In Turkey, epidemiological investigations are local, and the nationwide seroprevalence of toxoplasmosis and rubella is not documented.

The results of this study suggest that the prevention of CRS can be achieved through efficient vaccination programs. Rubella vaccination has been proven to be costeffective for the elimination of CRS.

Because there is a high seropositivity of rubella, the vaccination of children and preconceptional women is a better strategy for the prevention of CRS than the screening of pregnant women.

In our study, the seroprevalence for toxoplasma IgG was determined to be $25.5 \%$. Considering the low seroprevalence and serious fetal effects of congenital toxoplasmosis, antenatal screening seems worthwhile in the studied region.

Another issue is the lack of awareness among women of childbearing age of the risk factors of toxoplasmosis and rubella infection. The consumption of well-cooked meat, proper handling and disposal of fecal material of pet cats and elimination of oocysts from raw consumed food are important measures for the prevention of $T$. gondii infection. We should build public health strategies to educate women about the prevention of these infections.

We recommend the inclusion of rubella in the national vaccination program for schoolchildren and women of childbearing age in the preconceptional period. With the present data, we conclude that antenatal screening for toxoplasmosis is justified in the studied region.

\section{REFERENCES}

Aksit S, Egemen A, Ozacar T, Kurugol Z, Keskinoglu P, Tasbakan M, Caglayan S (1999). Rubella seroprevalance in an unvaccinated population in İmir: recommendations for rubella vaccination in Turkey. Pediatr. İnfect. Dis. J. 18:577-580.

Bar-Oz B, Levichek Z, Moretti ME, Mah C, Andreou S, Koren G (2004). Pregnancy outcome following rubella vaccination: a prospective controlled study. Am. J. Med. Genet. A. 130:52-57.

Cengiz SA, Cengiz L, Us E, Cengiz T (2005). Investigation of Rubella IgG and IgM antibodies with ELISA in pregnants' sera. Turk. J. Infect. 19:19-24.

Cherry $J$ (2004). Pediatrics infectious disease. $5^{\text {th }}$ ed. Saunders, Philadephia, USA Vol 2, pp. 2134-2137.

Doroudchi M, Dehaghani AS, Emad K, Ghaderi AA (2001). Seroepidemiological survey of rubella immunity among three populations in Shiraz, Islamic Republic of Iran. East Meditterr. Health J. 7:128-38.

Egerman RS, Beazley D (1998). Toxoplasmosis. Semin. Perinatol. 22:332.

Foulon W, Villena I, Stray-Pedersen B, Decoster A, Lappalainen M, Pinon JM, Jenum PA, Hedman K, Naessens A (1999). Treatment of toxoplasmosis during pregnancy: A multicenter study of impact on fetal transmission and children's sequelae at age 1 year. Am. J. Obstet. Gynecol. 180:410-415.

Gladwin M, Trattler B (2004). The Rest of RNA Viruses. In: Clinical Microbiolgy., $3^{\text {rd }}$ ed. MedMaster Inc., Miami, Florida, USA. pp. 215310

Gras L, Wallon M, Pollak A, Cortina-Borja M, Evengard B, Hayde M, Petersen E, Gilbert R (2005). Association between prenatal treatment and clinical manifestations of congenital toxoplasmosis in infancy: a cohort study in 13 European centres. Acta Paediatr. 94:1721-31.

Hossain A (1989). Seroepidemiology of rubella in Saudi Arabia. J. Trop. Pediatr. 35:169-170.

Lever AML, Ross MGR, Baboonian C, Griffiths PD (1987). Immunity to rubella among women of childbearing age. Br. J. Obstet. Gynecol. 94:208-212.

Liu Q, Wei F, Gao S, Jiang L, Lian H, Yuan B, Yuan Z, Xia Z, Liu B, Xu $X$, Zhu XQ (2009). Toxoplasma gondii infection in pregnant women in China. Trans. R. Soc. Trop. Med. Hyg. 103:162-166.

Mann JM, Preblud SR, Hoffman RE, Brandling-Bennet AD, Hinman AR, Herrmann KL (1981). Assessing risks of rubella infection during pregnancy. A standardized approach. JAMA 245:1647-1652.

MMWR (2001). Control and preventation of rubella: evaluation and management of suspected outbreaks, rubella in pregnant women, and surveillance for congenital rubella syndrome. MMWR Recomm. Rep. 50:1.

Remington JS, McLeod R, Thulliez P, Desmonts G (2006). Toxoplasmosis. In: Remington JS, Klein J (eds) Infectious Diseases of the Fetus and Newborn Infant $6^{\text {th }}$ ed. WB Saunders, Philadelphia, USA, pp. 947-1092.

Robertson SE, Cutts FT, Samuel R, Diaz-Ortega JL (1997). Control of rubella and congenital rubella syndrome (CRS) in developing countries. Part 2: vaccination against rubella. Bull. World Health Organ. 75:69-80.

Romand S, Wallon M, Franck J, Thulliez P, Peyron F, Dumon H (2001). Prenatal diagnosis using polymerase chain reaction on amniotic fluid for congenital toxoplasmosis. Obstet Gynecol. 97:296-300.

Seker S, Abasiyanik MF, Salih BA (2004). Rubella immune status of pregnant and non-pregnant women in Istanbul, Turkey. Saudi Med. J. 25:575-579. 
SYROCOT (Systematic Review on Congenital Toxoplasmosis) study group, Thiebaut R, Leproust S, Chene G, Gilbert R (2007). Effectiveness of prenatal treatment for congenital toxoplasmosis: a meta-analysis of individual patients' data. Lancet 369(9556):115-122.

Tang JW, Aarons E, Hesketh LM, Strobel S, Schalasta G, Jauniaux E, Brink NS, Enders G (2003). Prenatal diagnosis of congenital rubella infection in the second trimester of pregnancy. Prenat Diagn. 23:509512.

Topuzoglu A, Ozaydin GAN, Cali S, Cebeci D, Kalaca S, Harmanci H (2005). Assessment of sociodemograpfic factors and socio-economic status affecting the coverage of compulsory and private immunization services in Istanbul, Turkey. Public Health 119:862-869.

Uyar Y, Balci A, Akcali A, Cabar C (2008). Prevalence of rubella and cytomegalovirus antibodies among pregnant women in northern Turkey. New Microbiol. 31:451-455.
Wallon M, Kodjikian L, Binquet C, Garweg J, Fleury J, Quantin C, Peyron $F$ (2004). Long-term ocular prognosis in 327 children with congenital toxoplasmosis. Pediatrics 113(6):1567-1572.

Younes AT, Elian A, Darwish MA (1991). Rubella virus antibodies in women of childbearing age. J. Egypt Public Health Assoc. 66:397410.

Yücel A, Bozdayı B, Imir T (2002). Seroprevalence of TORCHE antibodies among pregnant women in Gazi University Hospital. Turk. J. Infect. 16(3):279-283. 